\title{
Multi-dye theranostic nanoparticle platform for bioimaging and cancer therapy
}

\author{
Amit K Singh ${ }^{1,2}$ \\ Megan A Hahn² \\ Luke G Gutwein ${ }^{3}$ \\ Michael C Rule ${ }^{4}$ \\ Jacquelyn A Knapik ${ }^{5}$ \\ Brij M Moudgil',2 \\ Stephen R Grobmyer ${ }^{3}$ \\ Scott C Brown 2,6 \\ 'Department of Materials Science and \\ Engineering, College of Engineering, \\ ${ }^{2}$ Particle Engineering Research Center, \\ College of Engineering, ${ }^{3}$ Division of \\ Surgical Oncology, Department of \\ Surgery, College of Medicine, ${ }^{4}$ Cell \\ and Tissue Analysis Core, McKnight \\ Brain Institute, ${ }^{5}$ Department of \\ Pathology, College of Medicine, \\ University of Florida, Gainesville, FL, \\ USA; ${ }^{6}$ DuPont Central Research and \\ Development, Corporate Center for \\ Analytical Science, Wilmington, \\ DE, USA
}

This article was published in the following Dove Press journal:

International Journal of Nanomedicine

31 May 2012

Number of times this article has been viewed

Background: Theranostic nanomaterials composed of fluorescent and photothermal agents can both image and provide a method of disease treatment in clinical oncology. For in vivo use, the near-infrared (NIR) window has been the focus of the majority of studies, because of greater light penetration due to lower absorption and scatter of biological components. Therefore, having both fluorescent and photothermal agents with optical properties in the NIR provides the best chance of improved theranostic capabilities utilizing nanotechnology.

Methods: We developed nonplasmonic multi-dye theranostic silica nanoparticles (MDT-NPs), combining NIR fluorescence visualization and photothermal therapy within a single nanoconstruct comprised of molecular components. A modified NIR fluorescent heptamethine cyanine dye was covalently incorporated into a mesoporous silica matrix and a hydrophobic metallonaphthalocyanine dye with large molar absorptivity was loaded into the pores of these fluorescent particles. The imaging and therapeutic capabilities of these nanoparticles were demonstrated in vivo using a direct tumor injection model.

Results: The fluorescent nanoparticles are bright probes (300-fold enhancement in quantum yield versus free dye) that have a large Stokes shift $(>110 \mathrm{~nm})$. Incorporation of the naphthalocyanine dye and exposure to NIR laser excitation results in a temperature increase of the surrounding environment of the MDT-NPs. Tumors injected with these NPs are easily visible with NIR imaging and produce significantly elevated levels of tumor necrosis (95\%) upon photothermal ablation compared with controls, as evaluated by bioluminescence and histological analysis.

Conclusion: MDT-NPs are novel, multifunctional nanomaterials that have optical properties dependent upon the unique incorporation of NIR fluorescent and NIR photothermal dyes within a mesoporous silica platform.

Keywords: bioluminescence, in vivo imaging, mesoporous silica nanoparticles, NIR fluorescence, photothermal ablation, theranostic

\section{Introduction}

A requirement for engineered theranostic nanoparticles (NPs) is the simultaneous ability to function as imaging contrast agents for disease localization and serve as mediators of disease therapy. For clinically relevant applications of these nanomaterials in oncology, optical components with absorption/emission in the near-infrared (NIR) (650-900 nm) are preferred candidates, due to their high detection sensitivity (fluorescent NPs) and their ability to deliver localized photothermal therapy (plasmonic NPs) at deeper penetration depths, due to the low extinction coefficients of water, tissue, and blood in this region. ${ }^{1}$

Theranostic NPs made of optical components incorporate a diagnostic agent (eg, fluorescent dye) with a photothermal therapeutic treatment (eg, plasmonic material).

Correspondence: Stephen R Grobmyer Division of Surgical Oncology,

Department of Surgery, University of Florida, Gainesville, FL 326 10, USA

Tel +l 3522650169

Fax +l 3522650262

Email stephen.grobmyer@surgery.ufl.edu 
A common example of a NIR fluorescent material as a diagnostic component is indocyanine green (ICG)encapsulated NPs. ${ }^{2}$ However, the inferior photophysical/ photochemical properties of ICG (low quantum yield [QY], low physiological stability, poor photostability under harsh synthesis conditions, and photobleaching) ${ }^{2}$ and technical challenges associated with incorporating it into an NP matrix leads to undesirable dye leaching and a lower signal-to-noise ratio in fluorescence imaging.

Having optical properties that are size and shape dependent, NIR plasmonic nanostructures are photothermal materials that have been widely explored for therapeutic applications..$^{3-5}$ However, a specific absorption profile may not be possible if a different size or shape is required for a particular application; in other words, these materials possess optical tunability but lack size/shape tunability for a given optical property. For example, in order to tune their plasmon resonances to the NIR, the size and geometry of gold NPs must be altered. This change in size and shape may affect their functionalization, uptake, transport, and targeting capabilities.

Combining plasmonic materials with a fluorescent component into a single theranostic nanoconstruct has numerous challenges and its use has remained limited thus far. ${ }^{6,7}$ The aforementioned limitations of fluorescent NPs can be further exacerbated by fluorescence quenching, due to the fluorophore's close proximity to the plasmonic component. ${ }^{8}$ Consequently, realization of optical image-guided therapy using theranostic NPs continues to be an area of active research.

Herein, we describe a simple approach for combining NIR fluorescent in vivo imaging and photothermal ablation therapy using mesoporous silica NPs as a carrier vehicle for multiple dyes. Mesoporous silica NPs have been explored to a great extent as imaging agents, drug carriers, and theranostic agents in various biological applications, most notably in oncology.,10 They are a common platform for theranostic nanomaterials, as the silica matrix can hold one agent while a different agent is introduced within the pores. Our multi-dye theranostic nanoparticles (MDT-NPs) are hybrid organic-inorganic nanoconstructs that employ multiple organic dyes for simultaneous imaging and cancer therapy. A mechanically robust scaffold of mesoporous silica NPs was selected for the synthesis of these hybrid NPs. A silica matrix is preferred due to its biocompatibility, optical transparency, and water dispersibility. In addition, Lee et al have found that mesoporous silica NPs exhibit less cytotoxicity and reduced inflammatory response and apoptosis than colloidal silica NPs of the same size and shape. ${ }^{11}$

\section{Materials and methods}

\section{Reagents}

IR780 iodide (IR780), 12-aminododecanoic acid, 3-aminopropyltriethoxysilane (APTES), 2-( $N$-morpholino) ethanesulfonic acid (MES), cetyltrimethylammonium bromide $\left(\mathrm{C}_{16} \mathrm{TAB}\right)$, silicon 2,3-naphthalocyanine dihydroxide (Si-naphthalocyanine), zinc 2,11,20,29-tetra-tert-butyl-2, 3-naphthalocyanine, iron (II) 1,2,3,4,8,9,10,11,15,16,17, 18,22,23,24,25-hexadecachloro-29H,31H-phthalocyanine, manganese (III) phthalocyanine chloride, and ICG were purchased from Sigma-Aldrich (St Louis, MO). Anhydrous $N, N$-dimethylformamide (DMF), triethylamine, $N$-hydroxysuccinimide (NHS), $N$-(3-Dimethylaminopropyl)$N^{\prime}$-ethylcarbodiimide hydrochloride (EDC), tetraethyl orthosilicate (TEOS), sodium hydroxide $(\mathrm{NaOH})$, methanol, calcium chloride $\left(\mathrm{CaCl}_{2}\right)$, and chloroform $\left(\mathrm{CHCl}_{3}\right)$ were purchased from Fisher Scientific (Pittsburgh, PA). Luciferin was purchased from Caliper Life Sciences (Hopkinton, MA).

\section{APTES-conjugated NIR fluorescent dye}

The NIR dye IR780 was chemically modified to include a silane group that covalently links the dye in the silica matrix (Figure S1). IR780 (300 mg) was dissolved in $10 \mathrm{~mL}$ of anhydrous DMF in a $100 \mathrm{~mL}$, round-bottom flask. Triethylamine $(200 \mu \mathrm{L})$ and 12-aminododecanoic acid $(254 \mathrm{mg})$ were added and the solution was heated for 3 hours at $85^{\circ} \mathrm{C}$ under a nitrogen environment. The green solution turned blue upon completion of the meso-chlorine replacement reaction. The carboxylic acid group of the resulting acid-conjugated dye was coupled with the primary amine of APTES, using carbodiimide coupling. Briefly, NHS (20 mg) was dissolved in $1 \mathrm{~mL}$ of acid-conjugated dye solution in DMF $(30 \mathrm{mg} / \mathrm{mL})$, after which EDC $(60 \mathrm{mg})$ dissolved in $400 \mu \mathrm{L}$ of $25 \mathrm{mM}$ MES buffer, pH 6.5, was added to the dye solution. After 15 minutes of stirring, $50 \mu \mathrm{L}$ of APTES was added to this solution, and the reaction proceeded for $\sim 2$ hours.

\section{Near-infrared fluorescence (NIRF) mesoporous silica NPs}

Mesoporous silica NPs were synthesized by condensation of TEOS in the presence of $\mathrm{C}_{16} \mathrm{TAB}$ as the structuredirecting template, under basic $\mathrm{pH}$ conditions at an elevated temperature. Briefly, $0.5 \mathrm{~g}$ of $\mathrm{C}_{16} \mathrm{TAB}$ and $1.75 \mathrm{~mL}$ of $2 \mathrm{M}$ $\mathrm{NaOH}$ were added to $240 \mathrm{~mL}$ of Nanopure ${ }^{\circledR}$ water (resistivity $18.2 \mathrm{M} \Omega-\mathrm{cm})$. This surfactant solution was heated to $80^{\circ} \mathrm{C}$. After the temperature stabilized, $2.5 \mathrm{~mL}$ of TEOS was added dropwise to this solution. After 2-3 minutes, the transparent solution turned translucent, due to the nucleation of silica NPs. 
At this time, the desired amount of APTES-conjugated fluorescent dye $(100-2000 \mu \mathrm{L})$ was added. The particle suspension was allowed to stir for 3 hours at $80^{\circ} \mathrm{C}$. To remove the surfactant, $\sim 800 \mathrm{mg}$ particles were centrifuged (20 minutes, $7000 \mathrm{~g}$ ) and redispersed in $200 \mathrm{~mL}$ of a $3 \%$ methanolic solution of $\mathrm{CaCl}_{2}$ then the suspension was refluxed for 4 hours at $60^{\circ} \mathrm{C}$. The NPs were centrifuged again (20 minutes, $\left.7000 \mathrm{~g}\right)$ and redispersed in methanol. This step was repeated three times, to remove the surfactant completely from the pores. Removal of surfactant was confirmed by a cell membrane permeability assay (Figure S2). Finally, the particles were dispersed in Nanopure water for further experiments.

\section{Incorporation of Si-naphthalocyanine heating dye}

NIRF mesoporous silica NPs (100 mg) were centrifuged and dispersed in $80 \mathrm{~mL}$ chloroform. Si-naphthalocyanine dye $(80 \mathrm{mg})$ in chloroform $\left(1 \mathrm{mg} \mathrm{mL} \mathrm{mL}^{-1}\right)$ was added to the silica NP dispersion. The dispersion was refluxed overnight at $60^{\circ} \mathrm{C}$, to load the dye into the pores of the NPs then the particles were centrifuged and redispersed in water for further experiments. The absorption spectrum of the supernatant was used to estimate the amount of dye that was adsorbed into the pores of the NPs.

\section{NP characterization}

Absorption and fluorescence spectra were obtained with a PerkinElmer LAMBDA 800 UV/VIS spectrometer (Waltham, MA) and a HORIBA Jobin Yvon NanoLog ${ }^{\circledR}$ fluorometer (Edison, NJ), respectively. Fluorescence QYs were measured using an integrating sphere to mitigate light scatter from the NPs, and the fluorescence and absorption of the sample were compared with the solvent blank. Scanning electron microscopy was performed with a JEOL JSM-6335F field emission scanning electron microscope (Tokyo, Japan) operating at $15 \mathrm{keV}$; transmission electron microscopy was performed with a JEOL 2010F transmission electron microscope operating at $200 \mathrm{keV}$. X-ray diffraction was obtained with a Philips APD 3720 X-ray diffractometer (Amsterdam, The Netherlands) using $\mathrm{Cu} \mathrm{K} \alpha$ radiation.

\section{Temperature change measurements}

A fiber-coupled continuous wave laser source (BWF2-785-5400-0.22-SMA; B\&W TEK Inc, Newark, DE) with a center wavelength of $785 \mathrm{~nm}$ was used. The output power of the laser was kept constant at $500 \mathrm{~mW}$ (450 mA laser diode current) in all experiments. An optical fiber sample cell was used to transfer the NIR laser radiation from the laser source to the sample cell. The end of the optical fiber was connected to a collimating lens and positioned $3 \mathrm{~cm}$ above the liquid level in the sample cell (Fisherbrand $1.5 \mathrm{~mL}$ methacrylate cuvette; Fisher Scientific). The sample cell was filled with a $1 \mathrm{~mL}$ aqueous suspension of mesoporous NPs. A thermocouple was fixed away from the laser path in the sample cell for temperature measurement.

\section{Experimental tumor model}

The 4T1 murine mammary tumor cell line expressing the firefly luciferase enzyme (Tufts University, Boston, MA) was orthotopically implanted $\left(1 \times 10^{6}\right.$ cells $)$ into female BALB/c mice (Charles River Laboratories, Wilmington, MA). ${ }^{12}$ On post-implantation day 8 , the mice were imaged via fluorescence (710 nm excitation/820 nm emission) and bioluminescence (620 nm emission, image obtained 10 minutes after intraperitoneal injection of luciferin $\left[100 \mu \mathrm{L}, 15 \mathrm{mg} \mathrm{mL}^{-1}\right]$ ) using an IVIS Spectrum (Caliper Life Sciences). The mice were randomly distributed to one of three groups - MDT-NP/+ablation, MDT-NP/-ablation, and saline/+ablation - and then underwent intratumoral injection of MDT-NPs $\left(1 \mathrm{mg} \mathrm{mL}^{-1}, 15 \mu \mathrm{L}\right)$ or $0.9 \% \mathrm{NaCl}(15 \mu \mathrm{L})$. Fluorescent imaging was obtained on all mice after the injection. The mice in the particular experimental groups then underwent photothermal ablative therapy (continuous wave laser, $785 \mathrm{~nm}, 637 \mathrm{~mW} \mathrm{~cm}^{-2}$, 5 minutes duration, $1.5 \mathrm{~cm}$ source-tumor distance), after which fluorescent imaging was obtained. All the mice then underwent bioluminescent imaging. On post-ablation day 1, the mice underwent fluorescent/bioluminescent imaging, after which they were euthanized. The tumors were harvested and preserved in $10 \%$ neutral buffered formalin for histological analysis. Histological tumor analysis was performed in a blinded fashion and quantified as percent necrosis by a boardcertified pathologist (JAK). Region of interest quantification of fluorescent and bioluminescent signals was performed at all imaging points with Living Image 3.2 software (Caliper Life Sciences). Approval for the animal studies was obtained from the University of Florida Institutional Animal Care and Use Committee and the experiments were all conducted in accordance with their policies.

\section{Results and discussion}

A reaction scheme for the synthesis of MDT-NPs is presented in Figure 1. First, a NIRF heptamethine cyanine dye was covalently incorporated within the silica framework during the synthesis of the mesoporous NPs, which prevents its leakage and enhances its photophysical properties by decreasing dye aggregation. The resulting NIRF NPs can be imaged with 

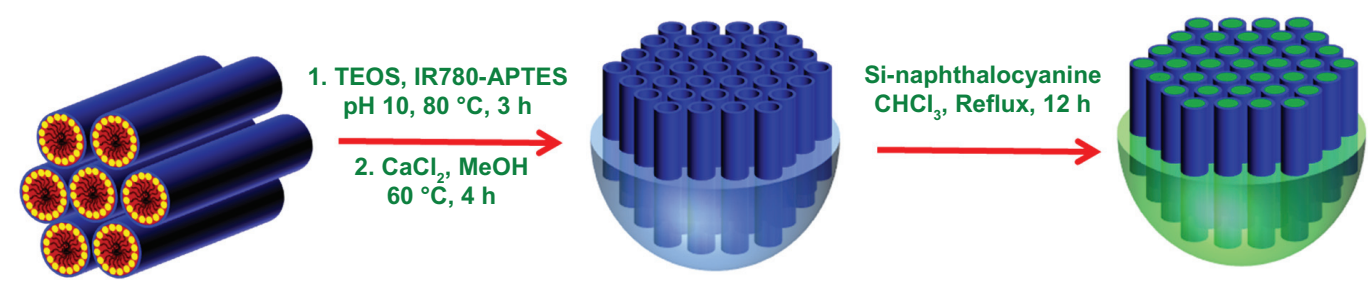

Figure I Reaction scheme for the synthesis of MDT-NPs. (Left) Hexagonal array of $\mathrm{C}_{16}$ TAB cylindrical micelles. (Center) NIRF silica nanoparticles formed by co-condensation of modified IR780-APTES dye conjugate with TEOS. (Right) MDT-NPs synthesized by encapsulation of Si-naphthalocyanine dye within the pores of mesoporous NIRF particles. Abbreviations: APTES, 3-aminopropyltriethoxysilane; $\mathrm{C}_{16}$ TAB, cetyltrimethylammonium bromide; DMF, N, N-dimethylformamide; IR780, IR780 iodide; MDT-NPs, multi-dye theranostic silica nanoparticles; NIRF, near-infrared fluorescence; TEOS, tetraethyl orthosilicate.

high signal-to-noise ratio, because their large Stokes shift $(>110 \mathrm{~nm})$ mitigates self-quenching and allows collection of the complete emission spectrum. Second, a NIR photothermal Si-naphthalocyanine dye was physically encapsulated in the cylindrical pores of the NIRF mesoporous silica NPs. The development of MDT-NPs from molecular-level components allows for the possibility of changing the size and shape of NPs without altering their optical properties. Different optical properties could be obtained simply by selecting dyes with the desired characteristics. Application of these theranostic MDT-NPs for bioimaging and therapy in a murine model of breast cancer is demonstrated. In order to overcome the aforementioned limitations of cyanine dyes, such as ICG, various modifications of these dyes have been developed to improve dye stability for NIR imaging. For example, heptamethine cyanine dyes have been modified by introducing a chlorocyclohexenyl ring in the methine chain, which improves photostability and fluorescence QY and provides a site for further chemical modification. ${ }^{13}$

In the present work, one of these heptamethine cyanine dyes (IR780) was modified with an aminoalkylcarboxylic acid; introducing an aminoalkyl group at the central position results in a relatively stronger fluorescent dye with a large Stokes shift $(>110 \mathrm{~nm})$ compared with IR780 itself. Due to this large Stokes shift, fluorescence resonance energy transfer among the dye molecules would be negligible, thus making encapsulation inside a solid matrix more viable while retaining high luminescence. This modified dye was further conjugated with an aminosilane, using carbodiimide chemistry in order to incorporate it covalently into the silica NPs during the hydrolysis and condensation of the silica precursor. Incorporating the dye molecules in surfactant micelles protects the dye from the harsh chemical environment commonly used in silica NP syntheses, where the NIR dye can lose its fluorescence properties. In addition, the micelles mitigate the quenching of fluorescence that often occurs because of dye aggregation in aqueous media during its incorporation within the silica matrix. A supramolecular self-assembly of mixed micelles composed of $\mathrm{C}_{16} \mathrm{TAB}$ and modified IR780APTES conjugate forms lyotropic liquid-crystalline phases that serve as the template for the formation of dye-doped, mesostructured silica NPs. Because of the well-dispersed (or evenly distributed) dye molecules in the silica matrix, the resulting NPs possess high photoluminescence and enhanced QY (300-fold increase over free dye), even in aqueous media (Figure S3). The shapes of the absorbance and fluorescence spectra and the large Stokes shift of the dye remain intact after its encapsulation in the particles. Normalized absorbance and emission spectra of the modified heptamethine dye in the free state and upon its encapsulation in mesoporous NPs are presented in Figure 2.

The QY of the heptamethine dye in the silica matrix was found to be 3.3-fold higher than the reported QY of ICG dye in a silica matrix (Figure $\mathrm{S} 3$ ). Increasing the amount of modified IR780 dye inside the silica matrix resulted in lower fluorescence QYs, most likely due to dye aggregation; however, the absorption cross-section and molar absorptivity of the NPs increased. The photoluminescence of these particles was optimized by varying the amount of dye present inside the silica matrix. The average size of these NIRF NPs was $105 \pm 16 \mathrm{~nm}$, as measured by scanning electron microscopy and transmission electron microscopy (Figure 3), with an average pore center-to-center distance of $3.6 \mathrm{~nm}$ (Figure S4).

To impart photothermal properties to NIRF NPs, an organic photothermal sensitizer can be incorporated within the pores of mesoporous NIRF NPs. The ideal candidate for this purpose should have low fluorescence QY and low photochemical reactivity for it to have good photothermal properties. After photoexcitation, these molecular absorbers are expected to increase the local temperature significantly enough to cause irreversible photothermal damage to the surrounding tumor cells. Naphthalocyanine dyes are particularly suited for this purpose, as they have an intense absorption band $\left(\varepsilon \approx 2 \times 10^{5} \mathrm{M}^{-1} \mathrm{~cm}^{-1}\right)$ in the NIR region, are nonfluorescent $(\mathrm{QY}<0.001)$, and lack appreciable biochemical activity. Many naphthalocyanine 


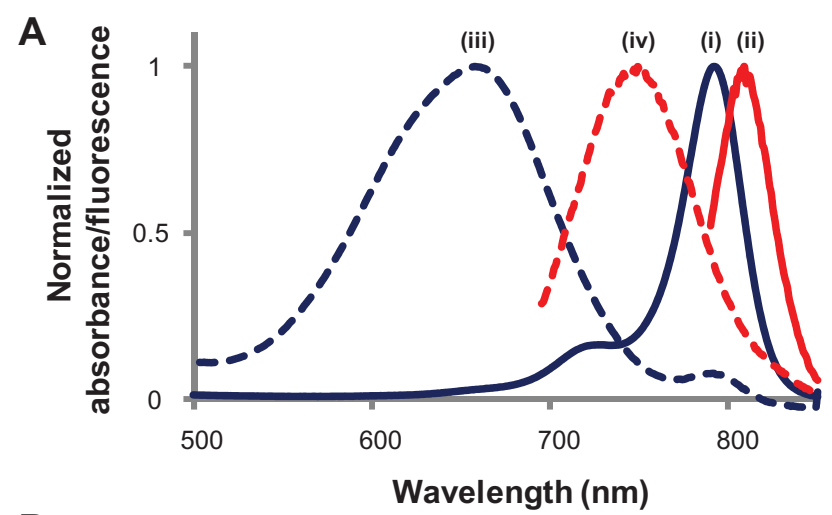

B

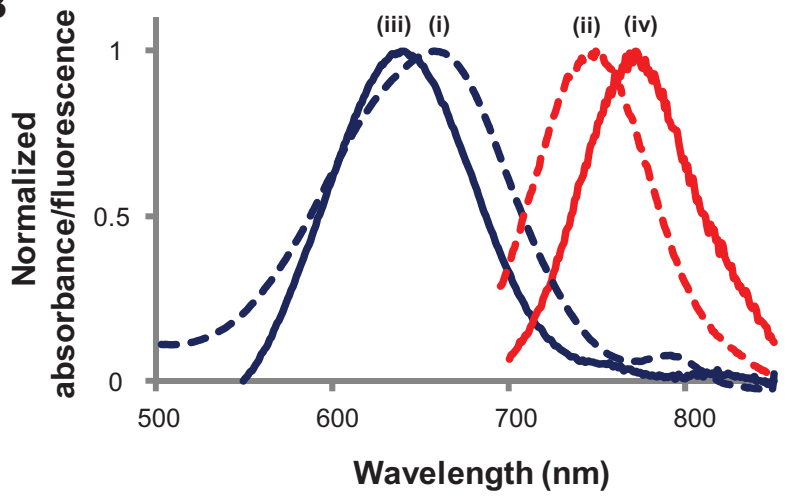

Figure 2 Normalized spectra: (A) IR780 dye before and after modification: (i) absorbance (IR780); (ii) fluorescence (IR780); (iii) absorbance (modified IR780); (iv) fluorescence (modified IR780). (B) Absorbance (iii) and fluorescence (iv) of modified IR780 dye after encapsulation in mesoporous silica nanoparticles.

Notes: Absorbance (i) and fluorescence (ii) of modified IR780 is also shown for comparison.

dyes are reported to be nonlinear optical materials that undergo triplet-state absorption at high laser fluencies, thereby enhancing light absorption-induced effects. ${ }^{14-17}$ At low light fluencies, such as those used for imaging, photoluminescence can be maximized due to reduced absorption from naphthalocyanine dyes. Furthermore, these materials are considered to be third-generation photodynamic agents and are actively being pursued for clinical applications: ${ }^{18}$ metallo-naphthalocyanine dyes have been shown to destroy cancer cells with a photothermal therapy effect, ${ }^{19,20}$ and they have been developed to act as photodynamic photosensitizers for cancer therapy by generation of singlet oxygen. ${ }^{21-23}$ This photodynamic effect can be synergistic with the proposed photothermal effect. Upon heating, and in the presence of a lipid-rich physiological environment, naphthalocyanine dye is believed to liberate itself from the particle and become regionally disseminated. During this process, the dye maintains some of its photothermal activity and may become more photodynamic in nature, similar to molecular sensitizers. As with the heptamethine cyanine dyes, incorporation of naphthalocyanine materials within a silica nanostructure was anticipated to provide the desired localized optical absorption properties for photothermal/ photodynamic effects, without the complications that are often experienced with free dye materials. Upon 2 minutes exposure to laser irradiation $\left(637 \mathrm{~mW} \mathrm{~cm}^{-2}\right)$, all metallo-(na) phthalocyanine dyes investigated here ( $\mathrm{Si}, \mathrm{Zn}, \mathrm{Fe}, \mathrm{Mn}$ ) either degraded or formed aggregates in aqueous media, due to their hydrophobic aromatic structure (Table S1). These aggregates can be up to several microns in size and are less effective for light absorption. Once encapsulated inside the particles, the photostability of the dye molecules increases drastically. Even after 5 minutes of $637 \mathrm{~mW} \mathrm{~cm}^{-2}$ laser light exposure, a maximum of only $5 \%$ decrease in the optical density of the NP suspension was observed.

In the present investigation, a Si-naphthalocyanine dye was loaded into the pores of NIRF mesoporous silica NPs by exposing them to a concentrated solution of dye that readily adsorbs within the pore structure. By using chloroform,

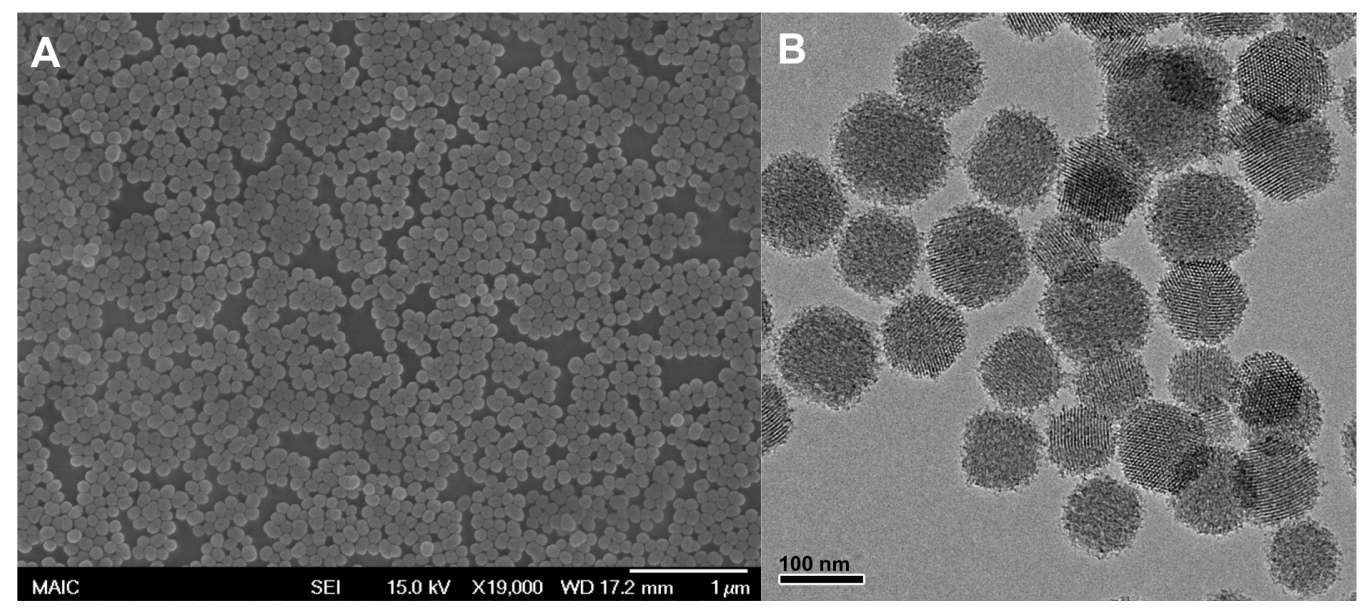

Figure 3 (A) Scanning electron microscopy and (B) transmission electron microscopy images of multi-dye theranostic silica nanoparticles. 
there is near refractive index matching between the dye solvent and the silica, which reduces London-van der Waal interactions and, therefore, dye-silica interactions, and promotes internal incorporation due to the dye concentration gradient. Incorporation of dye is confirmed by numerous observations, including: the NPs are still dispersible in water, despite the presence of the hydrophobic dye; fluorescence resonance energy transfer (which occurs at distances of $\sim 5 \mathrm{~nm}$ ) between the two dyes causes fluorescence quenching, indicating that the Si-naphthalocyanine dye is in close proximity to the fluorescent dye, which is incorporated within the silica matrix; and there is a broad peak in the NIR region of the electronic spectrum after extensive washing.

With an increased amount of Si-naphthalocyanine dye, the absorbance of the NPs increased, but the fluorescence intensity decreased (Figure 4A and B), because emitted photons from the NIR fluorescent dye were absorbed by Si-naphthalocyanine dye molecules; there is overlap between the fluorescence of the modified heptamethine cyanine dye and the absorption of the Si-naphthalocyanine dye.
(See UV-vis absorption spectrum of Si-naphthalocyanine dye, Figure S5.) Therefore, the ratio of fluorescent heptamethine cyanine dye to Si-naphthalocyanine dye within the mesoporous silica NPs was optimized (1:9 molar ratio) for the combined application of optical image-guided photothermal therapy, with a final fluorescent dye loading of $1.2 \%$ and a Si-naphthalocyanine dye loading of $11.1 \%$, by weight.

The photothermal properties of MDT-NPs were demonstrated by measuring the temperature increase of a NP suspension $(1 \mathrm{~mL})$ upon irradiation with a NIR laser source $(785 \mathrm{~nm}$, $637 \mathrm{~mW} \mathrm{~cm}^{-2}$, spot size [diameter] $=1 \mathrm{~cm}$ ). Figure $4 \mathrm{C}$ shows the increase in temperature of aqueous MDT-NP suspensions of different concentrations. After 5 minutes of irradiation time, a temperature change of $18^{\circ} \mathrm{C}\left( \pm 1.5^{\circ} \mathrm{C}\right)$ was measured for particles $\left(1 \mathrm{mg} \mathrm{mL}^{-1}\right)$ with $11 \%$ Si-naphthalocyanine dye $(\mathrm{w} / \mathrm{w})$ loading. Upon further increase of irradiation time, the suspension temperature did not change significantly (data not shown). There was no significant temperature change observed when Nanopure water alone was irradiated with the same laser source for the same duration of time.

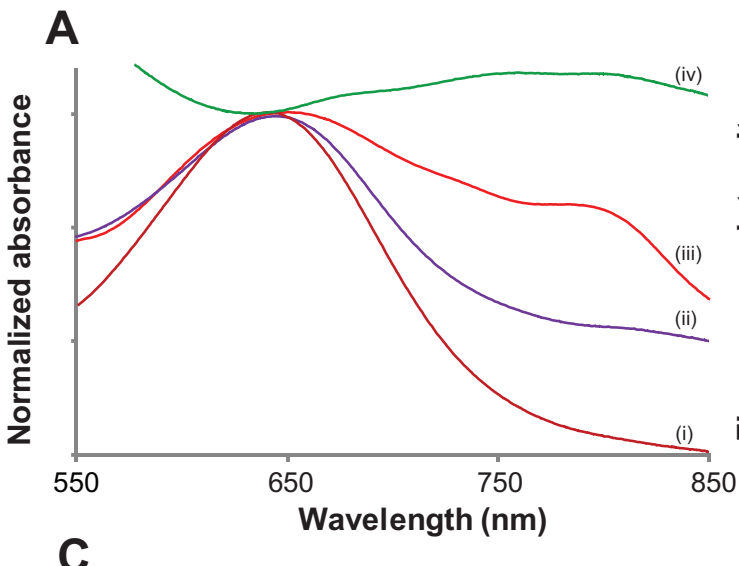

B
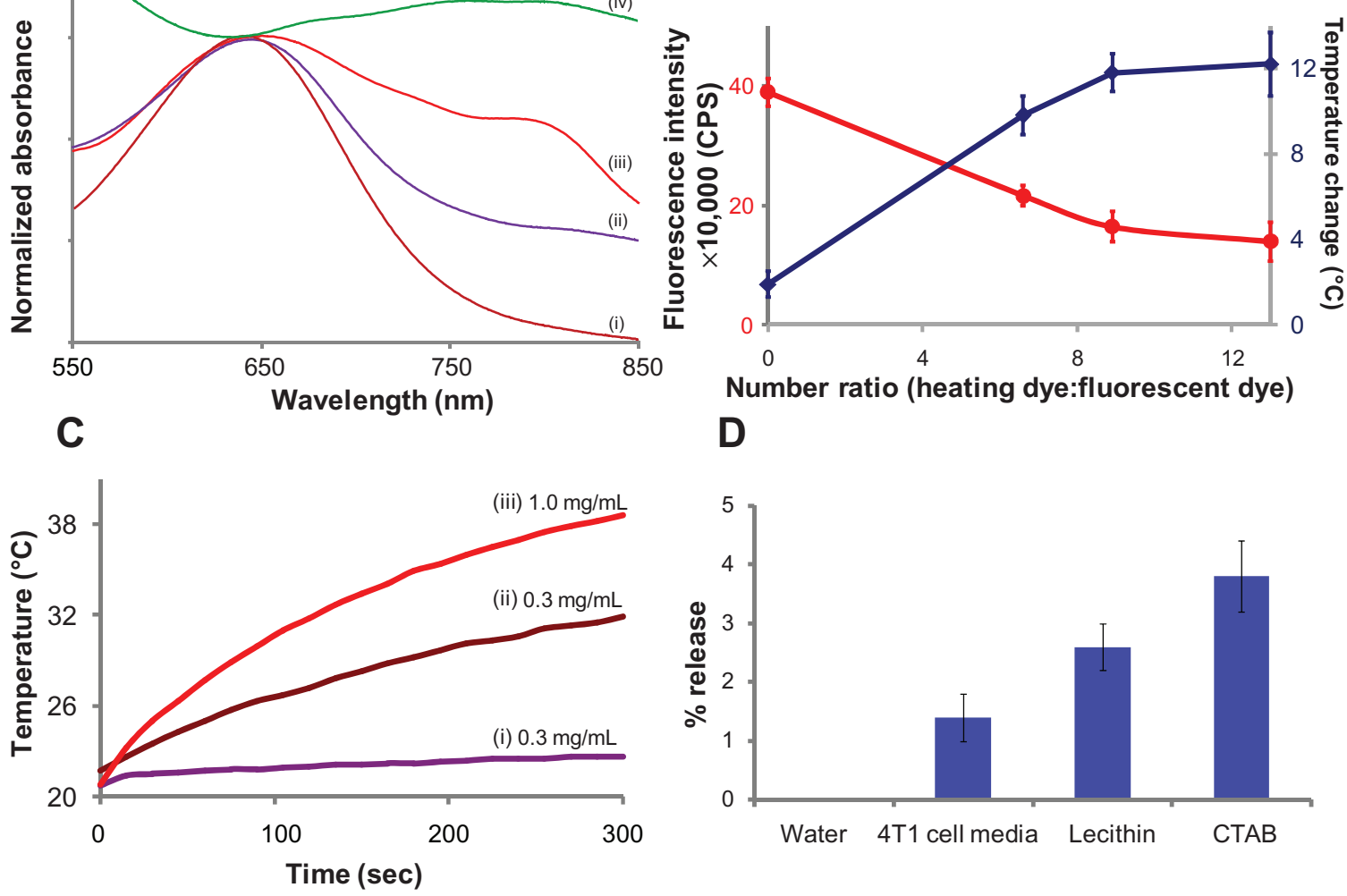

Figure 4 (A) Change in extinction of MDT-NPs upon increasing the amount of heating dye (Si-naphthalocyanine) encapsulated inside NIRF mesoporous silica NPs: (i) no Si-dye; (ii) 7.4\% Si-dye; (iii) II.1\% Si-dye; (iv) 14.5\% Si-dye. (B) Change in fluorescence and heating properties of MDT-NPs upon increasing the amount of Si-dye encapsulated inside NIRF mesoporous silica NPs. (C) Change in temperature of MDT-NP suspension in water when exposed to $637 \mathrm{~mW}^{\mathrm{cm}} \mathrm{cm}^{-2}$ laser irradiation for 5 minutes: (i) $[\mathrm{NP}]=0.3 \mathrm{mg} \mathrm{mL}-1$, no Si-dye; (ii) $[\mathrm{NP}]=0.3 \mathrm{mg} \mathrm{mL}-1$, I I. $1 \%$ Si-dye; (iii) $[\mathrm{NP}]=1.0 \mathrm{mg} \mathrm{mL}^{-1}$, I I. I\% Si-dye. (D) Release of dye as a percentage of initial dye loading (II.1\%) in different particle suspensions: water, 4TI cell media, lecithin $(50 \mathrm{mM}), \mathrm{C}_{16} \mathrm{TAB}(100 \mathrm{mM})$.

Abbreviations: $C_{16} T A B$, cetyltrimethylammonium bromide; MDT-NPs, multi-dye theranostic silica nanoparticles; NIRF, near-infrared fluorescence; NP, nanoparticle. 

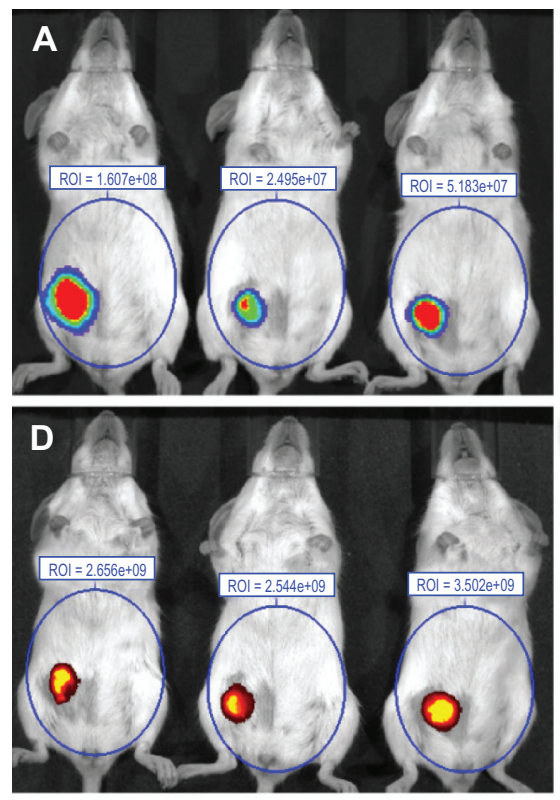

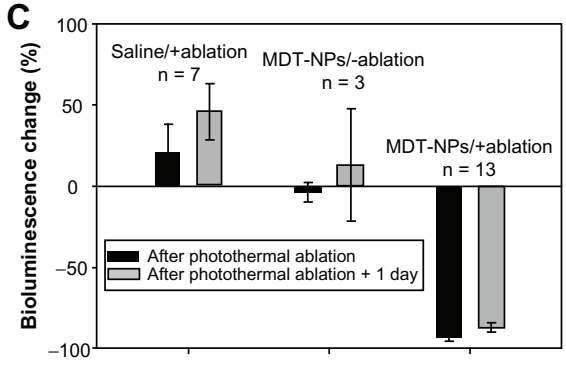

$\mathbf{F}$

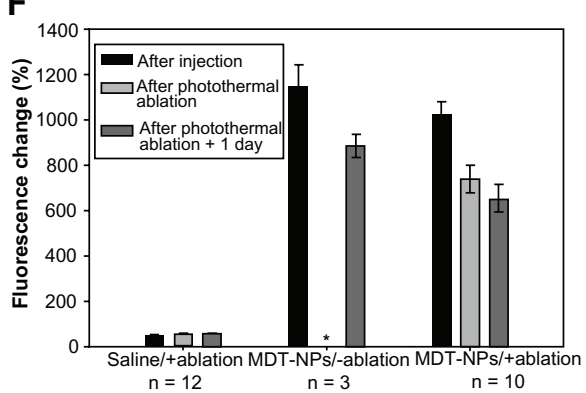

Figure 5 Bioluminescence ( $\mathbf{A}$ and $\mathbf{B}$ ) and fluorescence (D and $\mathbf{E})$ images of mice before (A and $\mathbf{D})$ and after (B and $\mathbf{E})$ photothermal ablation using a NIR laser (785 nm, $637 \mathrm{~mW} \mathrm{~cm}{ }^{-2}, 5$ minutes). All mice shown were treated with MDT-NPs/+ablation, and three were included to demonstrate reproducibility. Significant decrease in bioluminescence and little change in fluorescence signal intensity were observed after ablation. Change in $(\mathbf{C})$ bioluminescence signal and $(\mathbf{F})$ fluorescence before and after tumor ablation measured for saline/+ablation (left), MDT-NPs/-ablation (middle), and MDT-NPs/+ablation (right) at different time points. Y-axis represents percentage change from background signal that was measured prior to particle injection.

Note: *No fluorescence measured because no photoablation was performed.

Abbreviations: MDT-NPs, multi-dye theranostic silica nanoparticles; NIR, near-infrared; NIRF, near-infrared fluorescence; ROI, region of interest.

Due to the highly hydrophobic nature of the dye, a slight release in a media that can solubilize hydrophobic species is observed (Figure 4D). A slightly higher release may be expected in a biological environment, due to the presence of additional lipophilic species (eg, phospholipids, proteins, and serum), which can solubilize hydrophobic moieties.

In vivo application of MDT-NPs for image-guided therapy was demonstrated with an orthotopic murine mammary tumor model. This 4T1 tumor model was developed to provide immediate feedback to the photothermal therapeutic efficiency through in vivo imaging of bioluminescence, which is indicative of viable tumor cells. After intratumoral injection of MDT-NPs $\left(1 \mathrm{mg} \mathrm{mL}^{-1}, 15 \mu \mathrm{L}\right)$, the tumor was irradiated with the same low-power NIR laser source for 5 minutes. Figure 5A, B, D, and E present the fluorescence and bioluminescence images of tumor-bearing mice before and after the photothermal therapy. A decrease of $\sim 18 \%$ was observed in the fluorescence signal after laser irradiation. This decrease in fluorescence signal could be due to partial photobleaching of dye or slight particle redistribution during and after the tumor ablation. However, even one day after ablation, the fluorescence intensity remained $\sim 600 \%$ higher than the background signal (Figure 5F). A decrease in bioluminescence signal was calculated to assess tumor viability. A percentage decrease of up to $95 \%$ was observed in mice that were irradiated with the NIR laser after intratumoral injection of particles (Figure 5C). Minimal effects on bioluminescence were observed for control experiments (saline/+ablation and MDT-NPs/-ablation).

The exact mechanism of cell death in vivo is not known, although there is likely a contribution from the photothermal effect. Histologically, a congruent pattern was observed: saline/+ablation experiments resulted in tumor sections that were visibly viable, while MDT-NPs/+ablation showed significant areas of necrosis (Figure S6).

\section{Conclusion}

In summary, we have developed novel, hybrid organicinorganic MDT nanoconstructs by integrating multiple dyes within mesoporous silica NPs. First, a NIR heptamethine cyanine fluorescent dye was modified to enable its covalent incorporation into a mesoporous silica matrix. The photoluminescence of these NIRF NPs was optimized by minimizing the aggregation of dye molecules during their covalent integration. Second, a nonfluorescent metallonaphthalocyanine dye was incorporated within the pores of these particles in order to make them photothermal agents for therapeutic applications. Destruction of tumor cells using a semi-quantitative bioluminescence analysis of live mice agreed well with a histological analysis of tumor necrosis. 
In addition, the porous structure of these NPs enabled multiple functionalities, such as in vivo, multimodal imaging or site-specific photogated release of hydrophobic drug molecules using a NIR optical source. Based on a mesoporous silica platform, MDT-NPs can be personalized for a desired application, as individual components of this system can be tailored to obtain specific characteristics. Parameters that can be changed include the optical properties of the fluorescent and photothermal dye components, particle size, particle shape, pore size, and surface functionalization.

\section{Acknowledgments}

The authors acknowledge the financial support of the Particle Engineering Research Center at the University of Florida. This work was supported in part by the Department of Defense Breast Cancer Research Program (grant number 00085128), the National Science Foundation (grant number 00082755), and the Florida Department of Health BankheadColey Cancer Research Program. The transmission electron microscopy imaging was performed by Kerry Siebein at the Major Analytical Instrumentation Center, College of Engineering, University of Florida, which also houses the scanning electron microscope and X-ray diffractometer.

\section{Disclosure}

The authors report no conflicts of interest in this work.

\section{References}

1. Weissleder R. A clearer vision for in vivo imaging. Nat Biotechnol. 2001;19(4):316-317.

2. Altinoglu EI, Russin TJ, Kaiser JM, et al. Near-infrared emitting fluorophore-doped calcium phosphate nanoparticles for in vivo imaging of human breast cancer. ACS Nano. 2008;2(10):2075-2084.

3. Sohn K, Kim F, Pradel KC, et al. Construction of evolutionary tree for morphological engineering of nanoparticles. ACS Nano. 2009;3(8): 2191-2198.

4. Sau TK, Rogach AL. Nonspherical noble metal nanoparticles: colloidchemical synthesis and morphology control. Adv Mater. 2010;22(16): 1781-1804

5. El-Sayed MA. Small is different: shape-, size-, and composition-dependent properties of some colloidal semiconductor nanocrystals. Acc Chem Res. 2004;37(5):326-333.
6. Jin Y, Gao X. Plasmonic fluorescent quantum dots. Nat Nanotechnol. 2009;4(9):571-576.

7. Bardhan R, Chen W, Perez-Torres C, et al. Nanoshells with targeted simultaneous enhancement of magnetic and optical imaging and photothermal therapeutic response. Adv Funct Mater. 2009;19(24): 3901-3909.

8. Schneider G, Decher G, Nerambourg N, et al. Distance-dependent fluorescence quenching on gold nanoparticles ensheathed with layerby-layer assembled polyelectrolytes. Nano Lett. 2006;6(3):530-536.

9. Lee JE, Lee N, Kim T, et al. Multifunctional mesoporous silica nanocomposite nanoparticles for theranostic applications. Acc Chem Res. 2011;44(10):893-902.

10. Ambrogio MW, Thomas CR, Zhao YL, et al. Mechanized silica nanoparticles: a new frontier in theranostic nanomedicine. Acc Chem Res. 2011;44(10):903-913.

11. Lee S, Yun HS, Kim SH. The comparative effects of mesoporous silica nanoparticles and colloidal silica on inflammation and apoptosis. Biomaterials. 2011;32(35):9434-9443.

12. Tao K, Fang M, Alroy J, et al. Imagable $4 \mathrm{~T} 1$ model for the study of late stage breast cancer. BMC Cancer. 2008;8:228.

13. Peng XJ, Song FL, Lu E, et al. Heptamethine cyanine dyes with a large stokes shift and strong fluorescence: a paradigm for excited-state intramolecular charge transfer. J Am Chem Soc. 2005;127(12):4170-4171.

14. Nalwa HS, Kakuta A, Mukoh A. Third-order optical nonlinearities of tetrakis- $n$-pentoxy carbonyl metallo-naphthalocyanines. Chem Phys Lett. 1993;203(2-3):109-113.

15. Hanack M, Schneider T, Barthel M, et al. Indium phthalocyanines and naphthalocyanines for optical limiting. Coord Chem Rev. 2001; 219-221:235-258.

16. Chen Y, Hanack M, Araki Y, et al. Axially modified gallium phthalocyanines and naphthalocyanines for optical limiting. Chem Soc Rev. 2005;34(6):517-529.

17. Nalwa HS, Kobayashi S. Large third-order optical non-linearities of spin-cast thin films of novel metallo-naphthalocyanines. J Pept Res. 1998;2(1):21-30.

18. Allison RR, Sibata CH. Oncologic photodynamic therapy photosensitizers: a clinical review. Photodiagnosis Photodyn Ther. 2010;7(2): $61-75$.

19. Camerin M, Rello-Varona S, Villanueva A, et al. Metallo-naphthalocyanines as photothermal sensitisers for experimental tumours: in vitro and in vivo studies. Lasers Surg Med. 2009;41(9):665-673.

20. Camerin M, Rello S, Villanueva A, et al. Photothermal sensitisation as a novel therapeutic approach for tumours: studies at the cellular and animal level. Eur J Cancer. 2005;41(8):1203-1212.

21. Shopova M, Woehrle D, Mantareva V, et al. Naphthalocyanine complexes as potential photosensitizers for photodynamic therapy of tumors. J Biomed Opt. 1999;4(3):276-285.

22. Song LP, Li H, Sunar U, et al. Naphthalocyanine-reconstituted LDL nanoparticles for in vivo cancer imaging and treatment. Int $J$ Nanomedicine. 2007;2(4):767-774.

23. Ford WE, Rodgers MA, Schechtman LA, et al. Synthesis and photochemical properties of aluminum, gallium, silicon, and tin naphthalocyanines. Inorg Chem. 1992;31(16):3371-3377. 


\section{Supplementary information}

\section{Modification of IR780 dye}

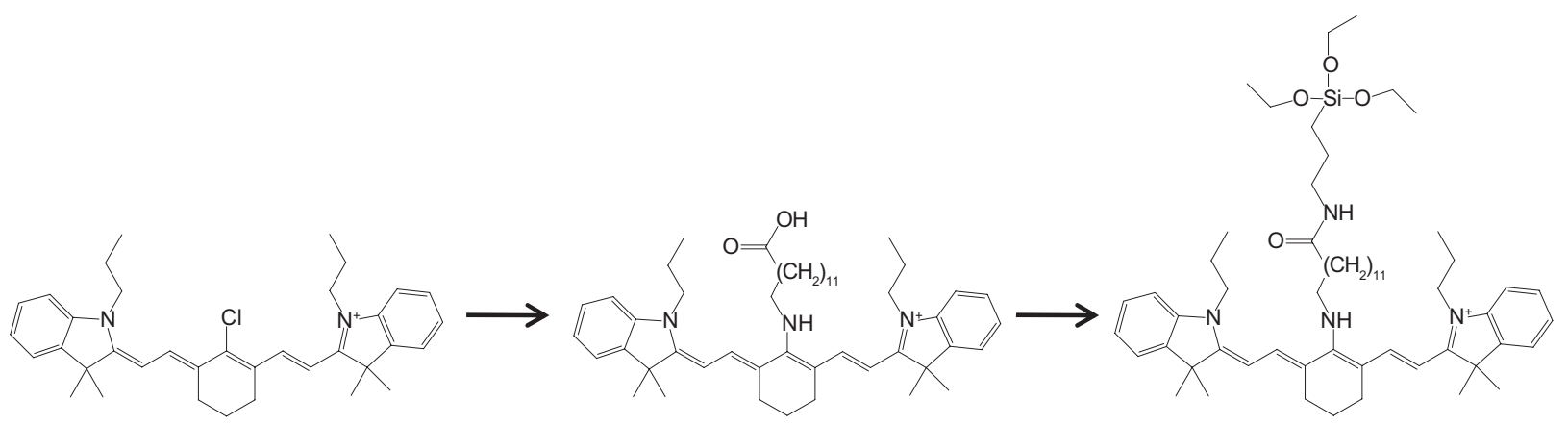

Figure SI Reaction scheme for the synthesis of modified NIR fluorescent IR780 dye. Synthetic procedures are described in Materials and methods. Abbreviations: APTES, 3-aminopropyltriethoxysilane; IR780, IR780 iodide; NIR, near-infrared.

\section{In vitro cytotoxicity}

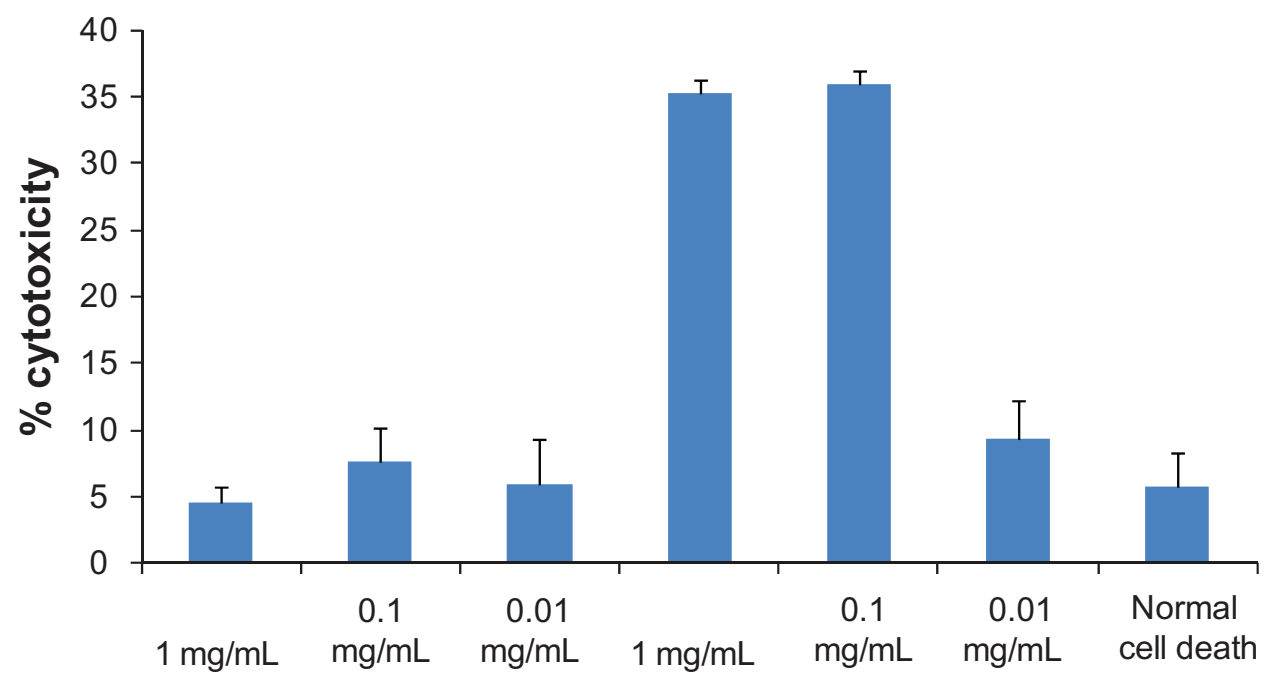

\section{Washed NPs}

\section{Unwashed NPs}

Figure $\mathbf{S 2}$ Effect of MDT-NPs on MDA-MDB-23I cells.

Notes: The MDT-NPs in water were incubated with MDA-MDB-23I breast epithelial cancer cells in culture and were demonstrated to be nontoxic by the lactate dehydrogenase membrane permeability assay following 24 hours exposure to NP concentrations up to I $\mathrm{mg} \mathrm{mL}^{-1}$. These results support the complete removal of the $C_{16}$ TAB surfactant from the mesoporous NP pores.

Abbreviations: $C_{16} T A B$, cetyltrimethylammonium bromide; MDT-NPs, multi-dye theranostic silica nanoparticles; NP, nanoparticle. 


\section{Comparison with ICG}
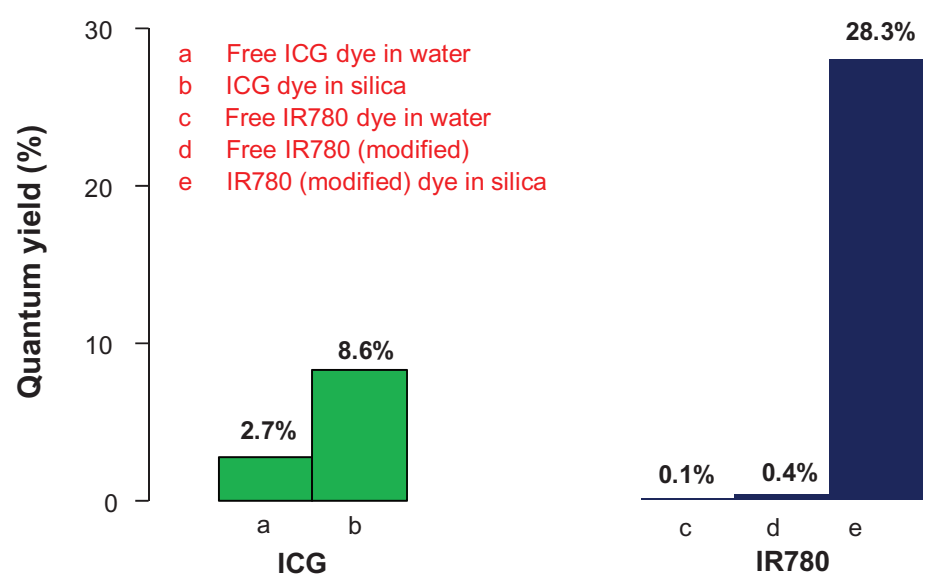

Figure S3 Quantum yield (QY) of IR780 dye increases upon its encapsulation in a mesoporous matrix.

Notes: A maximum enhancement of 283 -fold was observed when compared to free IR780 dye in water. The maximum QY of encapsulated IR780 also was compared with the QY of ICG in free state and encapsulated state (QY values for ICG were obtained from the literature).

Abbreviations: ICG, indocyanine green; IR780, IR780 iodide; qy, quantum yield.

\section{Pore size measurement}

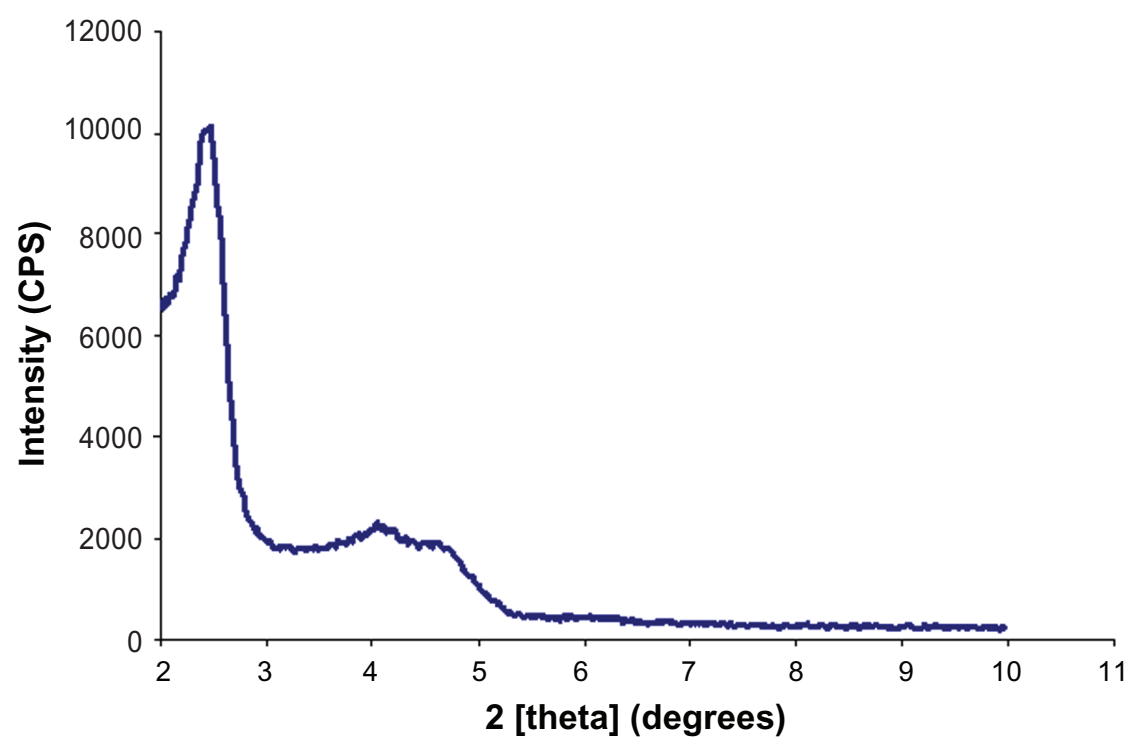

Figure S4 X-ray diffractometer pattern of near-infrared fluorescence mesoporous silica nanoparticles (x-axis: angle $2 \theta$ (degree), y-axis: intensity [CPS])

Note: The peak represents a pore center-to-center distance of $3.6 \mathrm{~nm}$, calculated using Bragg's equation.

Abbreviation: CPS, counts per second.

Table SI Effect of laser irradiation on different metallo-(na)phthalocyanine dyes in chloroform. All dyes were exposed to continuous laser excitation $\left(785 \mathrm{~nm}, 637 \mathrm{~mW} \mathrm{~cm}{ }^{-2}\right)$ for 2 minutes

\begin{tabular}{lllll}
\hline Dye & Conc $(\mathbf{m g} / \mathbf{m L})$ & Initial OD & Final OD & \% change \\
\hline $\mathrm{Si}$ & 0.13 & $\mathrm{I}$ & $0.05( \pm 0.02)$ & 95 \\
$\mathrm{Zn}$ & 0.01 & $\mathrm{I}$ & $0.06( \pm 0.02)$ & 94 \\
$\mathrm{Fe}$ & 0.03 & $\mathrm{I}$ & $0.06( \pm 0.01)$ & 95 \\
$\mathrm{Mn}$ & 0.08 & $\mathrm{I}$ & $0.05( \pm 0.01)$ & 96 \\
\hline
\end{tabular}

Notes: Si: silicon 2,3-naphthalocyanine dihydroxide (Si-naphthalocyanine); Zn: 2,II,20,29-tetra-tert-butyl-2,3-naphthalocyanine; Fe: iron (II) I,2,3,4,8,9,I0,II,15, I6, 17,18,22,23,24,25-hexadecachloro-29H,3|H-phthalocyanine; Mn: manganese (III) phthalocyanine chloride.

Abbreviations: Conc, concentration; OD, optical density. 


\section{Absorbance of Si-naphthalocyanine dye}

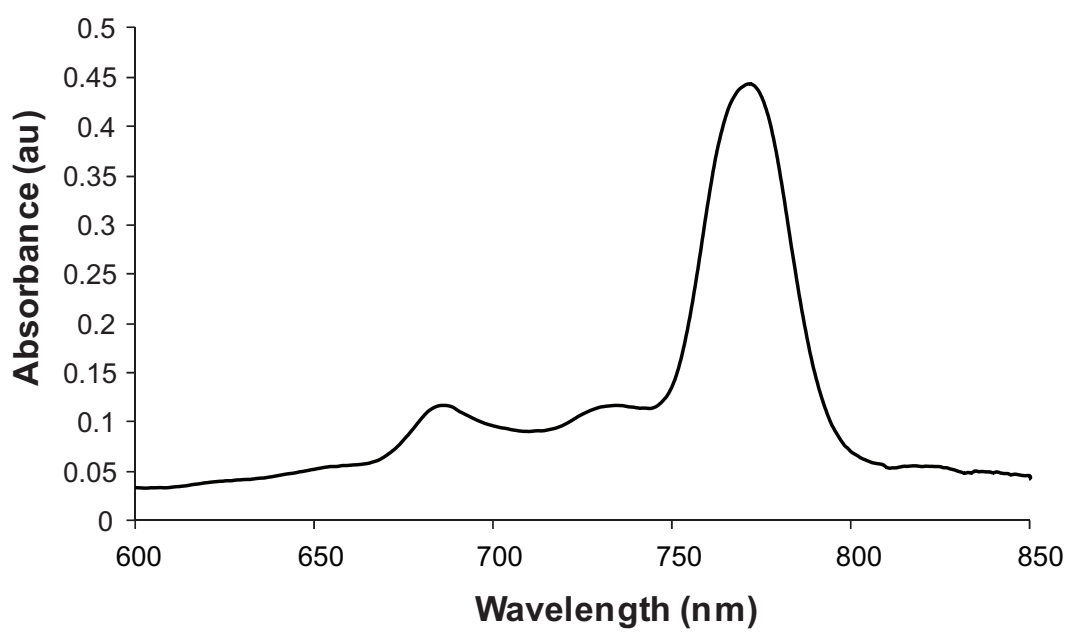

Figure S5 UV-vis absorbance spectrum of silicon 2,3-naphthalocyanine dihydroxide in dimethylformamide.

\section{In vivo experiments: histological analysis}

Figure S6A shows a viable tumor nodule for the saline/+ablation experiment, while Figure S6B demonstrates areas of necrosis, hemorrhage, and edema caused by localized heat generation of the MDT-NPs/+ablation set. In addition to in vivo imaging, MDT-NPs have the ability to be imaged in the visible region using confocal microscopy. With their broad and well- separated absorption and emission spectra, additional in vitro fluorescence assays are possible, such as flow cytometry for particle uptake studies and ex vivo histopathological analyses.
A

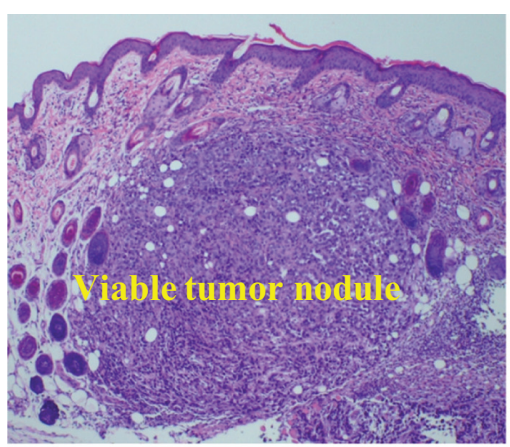

C

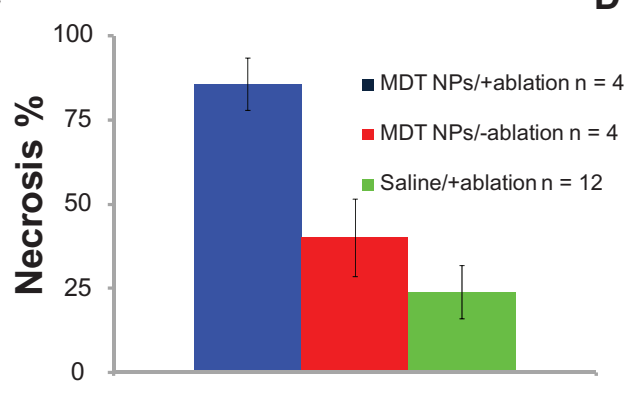

B

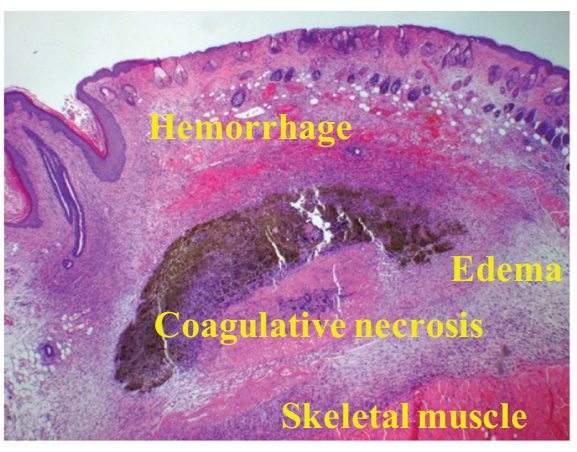

D

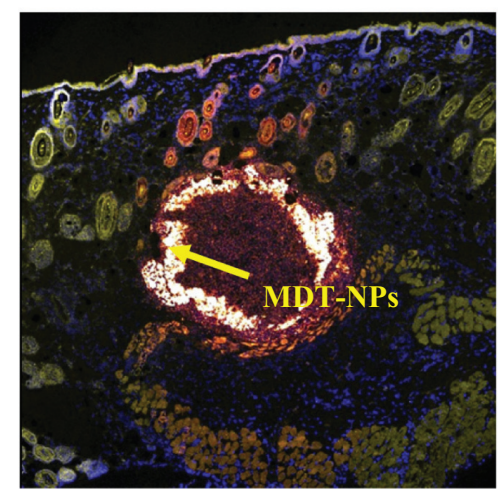

Figure S6 Histological analysis of tumor sections: (A) saline/+ablation and (B) multi-dye theranostic silica nanoparticles (MDT-NPs)/+ablation stained with hematoxylin and eosin demonstrate areas of viability and necrosis, respectively. (C) Histogram shows significantly more necrosis in tumors irradiated with laser in presence of MDT-NPs, as determined by a board-certified pathologist (JAK). (D) Confocal microscope image (I0× magnification) of a representative tissue section (stained using CellMaskTM Orange) containing MDT-NPs (shown by arrow). 


\section{Publish your work in this journal}

The International Journal of Nanomedicine is an international, peerreviewed journal focusing on the application of nanotechnology in diagnostics, therapeutics, and drug delivery systems throughout the biomedical field. This journal is indexed on PubMed Central, MedLine, CAS, SciSearch $\AA$, Current Contents ${ }^{\circledR} /$ Clinical Medicine,
Journal Citation Reports/Science Edition, EMBase, Scopus and the Elsevier Bibliographic databases. The manuscript management system is completely online and includes a very quick and fair peer-review system, which is all easy to use. Visit http://www.dovepress.com/ testimonials.php to read real quotes from published authors.

Submit your manuscript here: http://www.dovepress.com/international-journal-of-nanomedicine-journal 